\title{
High-stroke silicon-on-insulator MEMS nanopositioner: Control design for non- raster scan atomic force microscopy
}

Mohammad Maroufi, Anthony G. Fowler, Ali Bazaei, and S. O. Reza Moheimani

Citation: Review of Scientific Instruments 86, 023705 (2015); doi: 10.1063/1.4907908

View online: http://dx.doi.org/10.1063/1.4907908

View Table of Contents: http://aip.scitation.org/toc/rsi/86/2

Published by the American Institute of Physics

\section{Articles you may be interested in}

Note: A silicon-on-insulator microelectromechanical systems probe scanner for on-chip atomic force microscopy

Review of Scientific Instruments 86, 046107046107 (2015); 10.1063/1.4918729

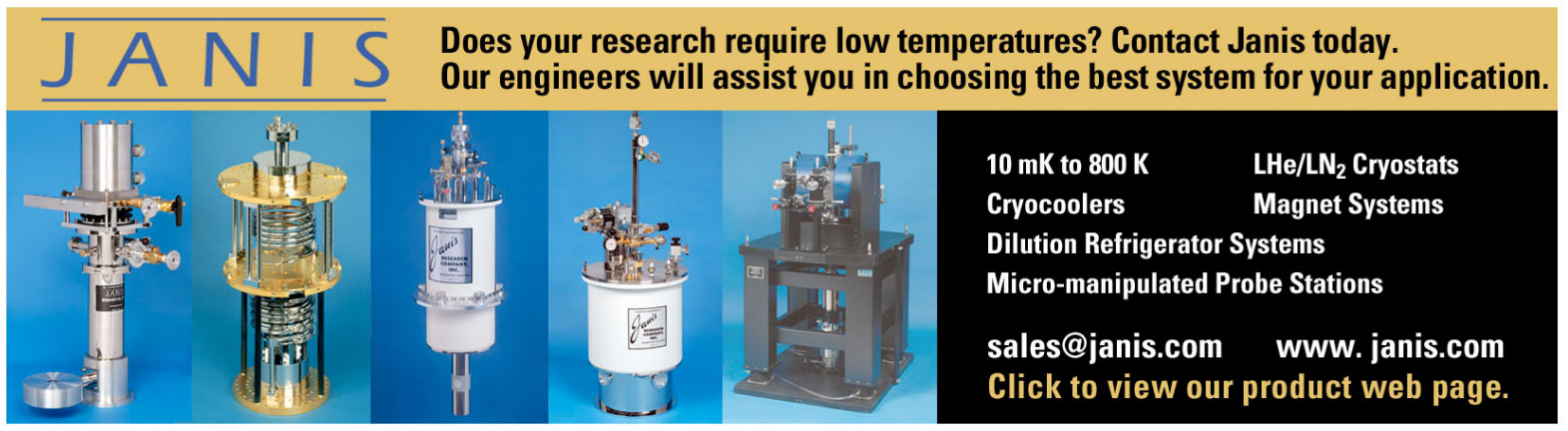




\title{
High-stroke silicon-on-insulator MEMS nanopositioner: Control design for non-raster scan atomic force microscopy
}

\author{
Mohammad Maroufi, ${ }^{\text {a) }}$ Anthony G. Fowler, ${ }^{\text {b) }}$ Ali Bazaei, ${ }^{c)}$ and S. O. Reza Moheimanid) \\ School of Electrical Engineering and Computer Science, University of Newcastle, \\ Callaghan NSW 2308, Australia
}

(Received 25 November 2014; accepted 29 January 2015; published online 17 February 2015)

\begin{abstract}
A 2-degree of freedom microelectromechanical systems nanopositioner designed for on-chip atomic force microscopy (AFM) is presented. The device is fabricated using a silicon-on-insulator-based process and is designed as a parallel kinematic mechanism. It contains a central scan table and two sets of electrostatic comb actuators along each orthogonal axis, which provides displacement ranges greater than $\pm 10 \mu \mathrm{m}$. The first in-plane resonance modes are located at $1274 \mathrm{~Hz}$ and $1286 \mathrm{~Hz}$ for the $\mathrm{X}$ and $\mathrm{Y}$ axes, respectively. To measure lateral displacements of the stage, electrothermal position sensors are incorporated in the design. To facilitate high-speed scans, the highly resonant dynamics of the system are controlled using damping loops in conjunction with internal model controllers that enable accurate tracking of fast sinusoidal set-points. To cancel the effect of sensor drift on controlled displacements, washout controllers are used in the damping loops. The feedback controlled nanopositioner is successfully used to perform several AFM scans in contact mode via a Lissajous scan method with a large scan area of $20 \mu \mathrm{m} \times 20 \mu \mathrm{m}$. The maximum scan rate demonstrated is 1 kHz. @ 2015 AIP Publishing LLC. [http://dx.doi.org/10.1063/1.4907908]
\end{abstract}

\section{INTRODUCTION}

Precise nanopositioning systems have proven to be integral to numerous scientific applications at the micro and nanoscale. These devices are able to provide highaccuracy mechanical displacements with nanometer and subnanometer resolution, typically with operating bandwidths of several hundred hertz or greater. These characteristics have led to their extensive utilization in applications including nanoassembly, ${ }^{1}$ optical beam steering, ${ }^{2}$ molecular biology, ${ }^{3}$ and nanolithography. ${ }^{4}$

Scanning probe microscopy is one of a multitude of applications in which high-performance nanopositioners play a key role. ${ }^{5}$ As a form of scanning probe microscopy, the atomic force microscope (AFM) ${ }^{6}$ is designed to measure the topography of a sample via a cantilever featuring a sharp tip of only a few nanometers in diameter. A nanopositioner integrated within the system is used to position the sample along a trajectory that allows the cantilever to be scanned across the entire surface of the sample, enabling a 3D image of the sample's topography to be generated.

Conventional nanopositioners are macroscale devices such as piezoelectric tube scanners or flexure-guided nanopositioners. $^{7-9}$ These devices are widely used in commercial AFMs, where they enable high-speed imaging with subnanometer resolution. Despite recent success in achieving high imaging speeds using this conventional macroscale technology, the high cost of commercial AFMs hinders their widespread use beyond specialized scientific applications.

\footnotetext{
a)Mohammad.Maroufi@uon.edu.au

b)Anthony.Fowler@uon.edu.au

c)Ali.Bazaei@newcastle.edu.au

d)Reza.Moheimani@newcastle.edu.au
}

Alternatively, there has recently been increasing utilization of microelectromechanical systems (MEMS) technology to develop microscale nanopositioners. ${ }^{10-13}$ Their implementation may provide significant benefits for existing systems, as well as facilitate the emergence of new applications. These miniaturized positioning devices potentially offer a number of significant advantages when compared with existing macroscale nanopositioners, including a greatly reduced volume, lower manufacturing costs, and simplified processes for bulk fabrication. ${ }^{14,15}$ These characteristics are achieved while maintaining useful positioning ranges and operating bandwidths, which have led to their use in novel applications such as probe-based data storage. ${ }^{16-18}$

In recent efforts, we have utilized the benefits of MEMS nanopositioners with the ultimate goal of developing an onchip AFM. As one of the major components of the AFM system, the miniaturization of the positioning stage is a significant step towards developing a fundamentally redesigned AFM that is both smaller and cheaper to manufacture than typical systems. This may increase its potential for use as a ubiquitous tool for scientific research and may additionally allow the AFM to inherit new uses as a portable characterization device or a cost-effective educational tool. A 2-degree-offreedom (DOF) MEMS nanopositioner was introduced in Ref. 19, with the device using a parallel kinematic design and electrostatic actuation. The nanopositioner achieved mechanical displacements in excess of $15 \mu \mathrm{m}$ per axis, and a bandwidth of approximately $820 \mathrm{~Hz}$. An AFM scan was performed to demonstrate the nanopositioner's potential utility, with the MEMS device being used (in open loop) as the sample scanning stage. An enhanced version of this MEMS nanopositioner was presented in Ref. 20, where electrothermal displacement sensors were added to the device. This enabled the system to be controlled in closed-loop, 
therefore improving the tracking accuracy of the device and allowing it to become resilient against the effect of external disturbances. The device was again demonstrated as the scan stage for an AFM, with a series of scans being performed using a scan window of $12.7 \mu \mathrm{m} \times 12.7 \mu \mathrm{m}$ at raster frequencies up to $100 \mathrm{~Hz}$.

This paper builds on the foundations of the previous work by presenting a new MEMS nanopositioner for onchip AFM that is designed to improve on the previous devices in a number of key areas. Electrostatic actuators are used as they are relatively straightforward to implement using conventional MEMS processes while offering superior actuation speeds compared with electrothermal actuators. ${ }^{21,22}$ The actuators are implemented using a new bidirectional layout that is designed to increase the nanopositioner's range of motion. This mechanism also reduces the effect of nonlinearities arising from the deflection of the flexures and allows a linear actuation mechanism to be implemented. ${ }^{23}$ This compensates for the quadratic voltage-displacement behavior typically associated with comb-finger electrostatic actuators, therefore simplifying the design of a closed-loop control scheme. Electrothermal sensors are integrated within the nanopositioner to measure mechanical displacements along each axis and facilitate feedback control of the stage. These sensors have sufficient bandwidth given the dynamics of the nanopositioner, and occupy a minimal footprint whilst being easily fabricated via standard MEMS processes. ${ }^{24}$

The AFM images obtained using the nanopositioner reported in Ref. 20 were performed using a raster scan pattern, whereby one axis tracks a triangular reference, while the other axis follows a slow ramp signal. However, there has been recent interest in the use of non-raster scan methods for AFM, which include the implementation of spiral, cycloid, and Lissajous scanning methods. ${ }^{25-29}$ The Lissajous pattern is constructed through the use of two pure tone sinusoidal signals with slightly differing frequencies or phases as the reference signal for each axis. It has been shown that this scan method provides a number of distinct advantages compared with raster scanning including significantly increased scanning speeds, improved image quality, and a simpler control implementation. $^{25}$ In contrast with other non-raster scan patterns, it also maintains the ability to produce an image using a complete square scan window. This paper demonstrates these benefits at the MEMS scale, with the Lissajous scan method being implemented for the designed MEMS nanopositioner.

The rest of the paper continues as follows. Section II describes the design of the nanopositioner and the electrothermal sensors. In Sec. III, the characterization and identification of the device are detailed. The Lissajous scan principle is briefly presented in Sec. IV, and the performance of the nanopositioner during an open-loop Lissajous scan is examined. Section V addresses the controller design and implementation for tracking a Lissajous trajectory. Finally, in Sec. VI, the performance of the controlled nanopositioner for AFM imaging is investigated at different scan frequencies.

\section{NANOPOSITIONER DESIGN}

The proposed nanopositioner is an improved version of the device reported in Ref. 20. As shown in the scanning electron microscope (SEM) image of the device in Fig. 1, the nanopositioner uses a parallel kinematic mechanism to produce motion in two orthogonal directions. The standard SOIMUMPs process provided by MEMSCAP is used for fabrication, ${ }^{30}$ with $25 \mu$ m-thick single crystal silicon being used as the structural material (Table I). As an improvement in this design, electrostatic actuators are implemented adjacent to each side of the central scan table, which permit bidirectional motion along both axes. In addition to allowing a larger displacement range, the bidirectional actuators make a linear actuation mechanism feasible for the device, which mitigates the quadratic nonlinearity typically associated with electrostatic actuation and makes the control of the device more straightforward. ${ }^{23}$

The device features a $3 \mathrm{~mm} \times 3 \mathrm{~mm}$ scan table located at the center, and clamped-clamped beams (types A and B) are used as the mechanical suspension. U-shaped shuttles are implemented on each side of the stage, which provide a base for the actuating electrostatic combs. The generated force from the electrostatic actuators is transferred to the scan table by the U-shaped shuttle and ten flexures (type B) on each side
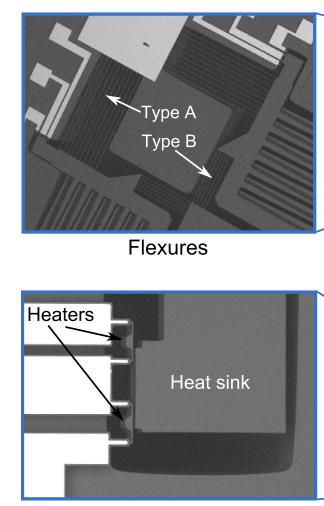

Electrothermal sensor
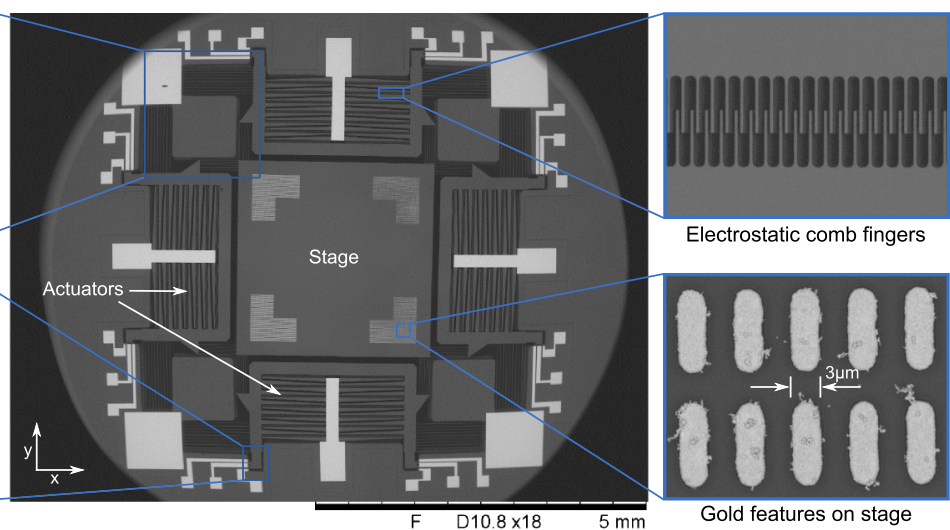

FIG. 1. SEM images of the fabricated nanopositioner, with close-up views of the electrostatic actuators, gold features, electrothermal sensors, and flexures. The gold features have a length of $9 \mu \mathrm{m}$ and a width/spacing of $3 \mu \mathrm{m}$. 
TABLE I. Geometric and material properties of the nanopositioner. The number of flexures and comb structures is reported per side of the device.

\begin{tabular}{|c|c|c|c|c|}
\hline \multicolumn{3}{|c|}{ Density: $2500\left(\mathrm{Kg} / \mathrm{m}^{3}\right)$} & \multicolumn{2}{|c|}{ Young's modulus: $169 \mathrm{GPa}$} \\
\hline \multirow{3}{*}{ Flexure type } & & Length & Width & Number \\
\hline & A & $1100 \mu \mathrm{m}$ & $5 \mu \mathrm{m}$ & 20 \\
\hline & $\mathrm{B}$ & $600 \mu \mathrm{m}$ & $5 \mu \mathrm{m}$ & 10 \\
\hline Comb fingers & \multicolumn{4}{|c|}{$\begin{array}{l}\text { Gap: } 2 \mu \mathrm{m}, \text { Engagement: } 13 \mu \mathrm{m} \text {, } \\
\text { Length: } 30 \mu \mathrm{m}, \text { Width: } 2 \mu \mathrm{m}\end{array}$} \\
\hline Comb structures & \multicolumn{4}{|c|}{$\begin{array}{l}\text { Length: } 1000 \mu \mathrm{m} \text {, Short base: } 20 \mu \mathrm{m} \text {, } \\
\text { Long base: } 60 \mu \mathrm{m} \text {, No. per side: } 14\end{array}$} \\
\hline U-shaped structure & \multicolumn{4}{|c|}{$\begin{array}{c}\text { Length: } 2600 \mu \mathrm{m}, \text { Width: } 187 \mu \mathrm{m} \text {, } \\
\text { Side length: } 1234 \mu \mathrm{m}\end{array}$} \\
\hline Total size & \multicolumn{4}{|c|}{$6.6 \mathrm{~mm} \times 6.6 \mathrm{~mm}$} \\
\hline
\end{tabular}

(Fig. 1). These beams are designed close to the edge of the scan table to prevent any in-plane rotation, resulting in significant cross coupling reduction.

The dimensions of the flexure beams are chosen such that they provide enough stiffness to achieve the desired resonance frequency, while maintaining a suitable displacement range in each direction. Further explanations regarding the calculation of the clamped-clamped beams' stiffness and their mass contribution to the in-plane resonance mode of the stage are provided in Refs. 23 and 31. During the dimensional tuning, we also reduced the mass of the electrostatic actuators using a trapezoidal beam profile for the comb structures. To demonstrate the performance of the device under an AFM, gold features are also fabricated on the scan table, which are used as the reference pattern, as illustrated in Fig. 1. The material and geometric properties of the device are reported in Table I.

To estimate the resonance frequency of the stage, a finite element model (FEM) is constructed in CoventorWare software. The modal analysis reveals a resonance frequency of about $1479 \mathrm{~Hz}$ for the first in-plane mode for both axes (Fig. 2).

\section{A. Electrothermal sensors}

To measure the displacement of the stage in both axes, electrothermal sensors are implemented in each direction as shown in the close-up view in Fig. 1. The U-shaped

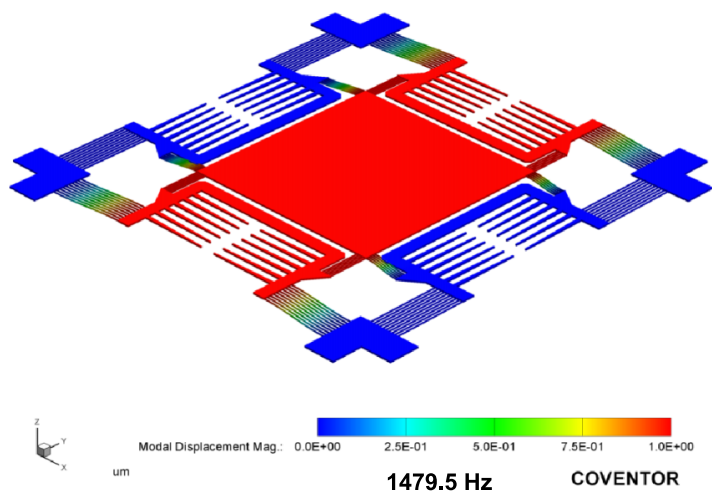

FIG. 2. The first in-plane resonance mode of the device obtained by FEM. shuttles are extended slightly and are used as heat sinks for resistive heaters in the sensors. These heaters feature a contoured profile, which has been shown to improve their linearity. ${ }^{32}$ As the equilibrium position of the heat sink is located halfway between the two heaters, the displacement of the heat sink creates a differential temperature variation between the heaters. The change of temperature leads to a proportional variation in their electrical resistance. Using a V-I transimpedance readout circuit, the resistance difference between the heaters is mapped to the displacement of the heat sink. As illustrated in Fig. 3, the readout circuit is comprised of two stages. A bias voltage $\left(V_{c c}\right)$ heats up the beams electrothermally and the transimpedance amplifiers in the first stage convert the heater currents to proportional voltages. The second stage is the differential amplifier, which amplifies the difference between the two voltages. ${ }^{15,33}$

\section{DEVICE CHARACTERIZATION AND IDENTIFICATION}

To characterize the nanopositioner, a 3D Polytec Micro System Analyzer (MSA) was used. This device exploits three laser vibrometers to measure and record the static and dynamic behavior of the stage. To implement the linear actuation mechanism for each axis, a differential actuation voltage plus a $40 \mathrm{~V}$ DC bias voltage are applied to the opposing stator combs while the rotor combs are connected to the electrical ground. Here, the net force exerted on the stage is linearly related to the magnitude of the differential actuation voltage and the DC bias. ${ }^{23}$ Fig. 4 shows the displacement of the scan table versus the differential actuation voltage. The results illustrate that the stage has an almost linear behavior and a displacement range of more than $\pm 10 \mu \mathrm{m}$ for both axes. The figure also shows the output voltages of the electrothermal sensors for both axes, which were recorded simultaneously. Using the actual displacement and the corresponding sensor output reported in Fig. 4, calibration factors of $0.729 \mathrm{~V} / \mu \mathrm{m}$ and $0.731 \mathrm{~V} / \mu \mathrm{m}$ were obtained for the $\mathrm{X}$ and $\mathrm{Y}$ axes, respectively.

To obtain the frequency response of the system in the open-loop condition, a chirp signal is applied differentially

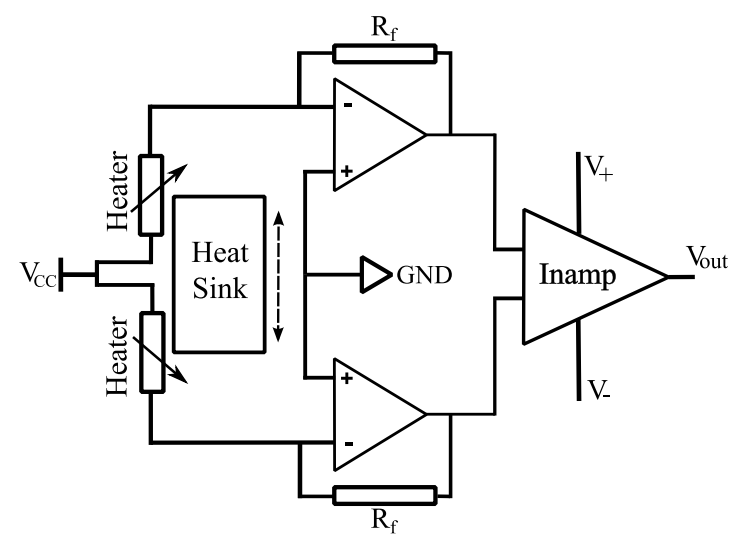

FIG. 3. The schematic of the electrothermal sensors' readout circuit using a V-I transimpedance mechanism. 


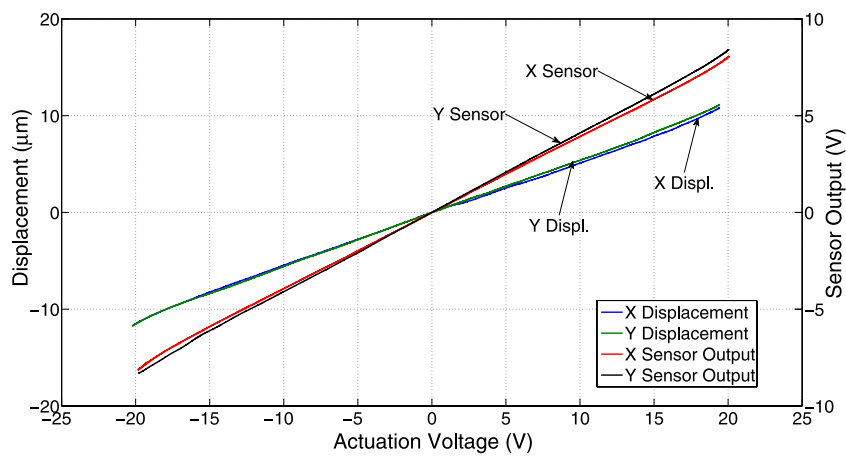

FIG. 4. The stage displacement (left-hand axis) and sensor outputs (righthand axis) due to an applied differential actuation voltage for both axes.

to the device through the same linear actuation mechanism, which leads to small displacements of the scan table around its equilibrium position. The displacement of the scan table was measured by the MSA, with the electrothermal sensor signal being recorded simultaneously. The frequency response of the system obtained by the MSA and sensors are compared in Fig. 5 for both axes. This comparison shows that the electrothermal sensors satisfactorily capture the dynamics of the systems in both axes. The resonance frequencies of the stage are measured to be $1274 \mathrm{~Hz}$ for the $\mathrm{X}$ axis and $1286 \mathrm{~Hz}$ for the $\mathrm{Y}$ axis, which are more than $30 \%$ higher than the previous version. ${ }^{20}$ The inconsistency which can be observed between the experimental and FEM results is most likely due to the tolerances of the fabrication process.
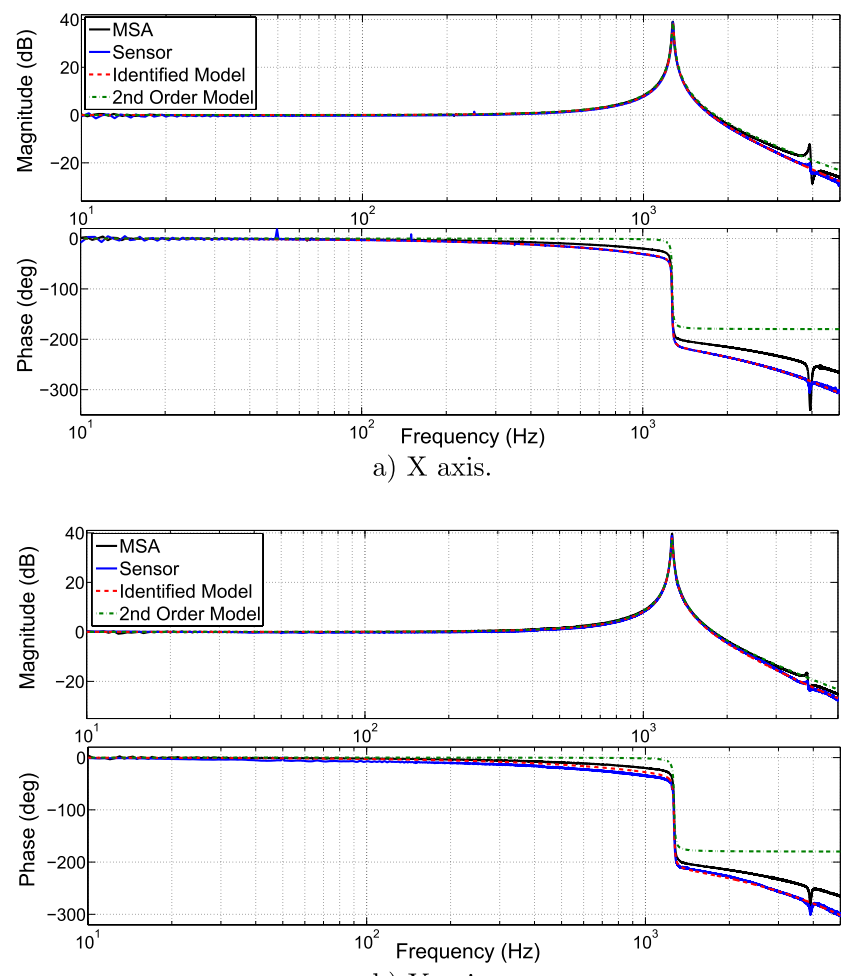

b) $\mathrm{Y}$ axis.

FIG. 5. Frequency response of the system in the $\mathrm{X}$ and $\mathrm{Y}$ axes obtained by MSA and electrothermal sensor. The frequency responses for the identified model and the estimated second order model are also shown.
The cross coupling between the two axes of motion is experimentally obtained by measuring the displacement induced in one axis resulting from large-amplitude actuation of the orthogonal axis. This leads to a cross coupling measurement of approximately $-40 \mathrm{~dB}$ for both axes. The cross coupling rejection for this device is comparable with (and in some instances better than) those reported previously in the literature, which range from $-35 \mathrm{~dB}$ to $-40 \mathrm{~dB} .^{34}$

\section{A. System identification}

The least squares method in the frequency domain is applied for system identification. ${ }^{35}$ Due to the negligible cross coupling between the two orthogonal axes, the dynamics of the nanopositioner in each direction can be accurately considered as a single-input single-output (SISO) system. Following third order models (1) completely capture the dynamics of the system for both axes in the bandwidth of interest (Fig. 5),

$$
\begin{aligned}
G_{x x} & =\frac{1.011 \times 10^{-9} s^{2}-5.2 \times 10^{-5} s+1.604}{7.666 \times 10^{-13} s^{3}+1.57 \times 10^{-8} s^{2}+5.04 \times 10^{-5} s+1}, \\
G_{y y} & =\frac{1.015 \times 10^{-9} s^{2}-5.349 \times 10^{-5} s+1.638}{6.566 \times 10^{-13} s^{3}+1.586 \times 10^{-8} s^{2}+4.31 \times 10^{-5} s+1} .
\end{aligned}
$$

\section{LISSAJOUS SCAN PRINCIPLE AND OPEN-LOOP TRACKING}

Typically, the raster scanning method is implemented for nanopositioning purposes in various types of scanning probe microscopes. In this method, the positioning stage follows a triangle or sawtooth waveform in one axis. ${ }^{7,36}$ However, in addition to the fundamental frequency, a triangle or sawtooth waveform contains significant harmonics which will induce vibrations in the nanopositioner and effectively limit the achievable scan speed and tracking accuracy of the stage. Using the raster scan method in open loop, a scan frequency of approximately 1/100th of the device's dominant resonance frequency is achievable, ${ }^{7}$ however, this frequency can be increased by the implementation of closed-loop feedback controllers. In the previous device, ${ }^{20}$ a maximum raster frequency of $100 \mathrm{~Hz}$ was achieved. Given the higher bandwidth of the current device, a raster frequency of approximately $149 \mathrm{~Hz}$ could be expected for this nanopositioner assuming similar control loop performance. However, the implementation of the Lissajous scanning method allows the scan speed to be increased significantly further above this value. ${ }^{25,27}$

The Lissajous pattern is obtained in this case by driving the orthogonal axes of the nanopositioner with sinusoidal signals of different frequencies $\left(f_{x}\right.$ and $\left.f_{y}\right)$ and identical phase shifts $\left(0^{\circ}\right)$. In (2), the actuation signals $V_{x}$ and $V_{y}$ are presented for the $\mathrm{X}$ and $\mathrm{Y}$ axes, respectively. To scan a square-shaped area, the amplitudes of the actuation signals $\left(A_{x}\right.$ and $\left.A_{y}\right)$ should be tuned to obtain similar displacement amplitudes in both directions,

$$
\begin{aligned}
& V_{x}=A_{x} \sin \left(2 \pi f_{x} t\right) \\
& \quad \text { and } \\
& V_{y}=A_{y} \sin \left(2 \pi f_{y} t\right) .
\end{aligned}
$$




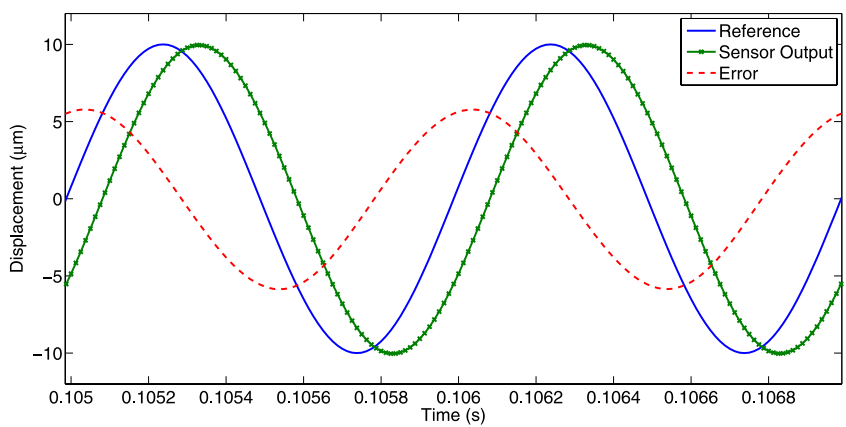

FIG. 6. Tracking performance of the nanopositioner's $\mathrm{X}$ axis in open loop. The reference is a $1 \mathrm{kHz}$ sinusoidal signal with an amplitude of $10 \mu \mathrm{m}$.

One of the frequencies (e.g., $f_{x}$ ) can be chosen based on the nanopositioner bandwidth, and the other frequency (here, $f_{y}$ ) can be obtained by (3),

$$
f_{y}=\frac{2 N-1}{2 N} f_{x} .
$$

In (3), the integer number $\mathrm{N}$ is related to the resolution and the amplitude of the sinusoidal signal as

$$
N=\text { Round }\left(\frac{\pi A_{x} A_{y}}{h \sqrt{A_{x}^{2}+A_{y}^{2}}}\right),
$$

where $(h)$ is the resolution needed for the scan which can be estimated based on the intended number of pixels in the image. The minimum time required for the Lissajous pattern to scan the entire area is

$$
t_{\text {scan }}=\frac{1}{2\left(f_{x}-f_{y}\right)}=\frac{N}{f_{x}} .
$$

To capture all required points, the sampling rate should be greater than a minimum required value provided in (6),

$$
F_{s, \min }=4 N(2 N-1)\left(f_{x}-f_{y}\right) .
$$

The sampling rate is obtained based on the minimum time interval between two successive crossing points in the trajectory. ${ }^{25}$

To examine the open-loop performance of the nanopositioner, the system was used to track a Lissajous scan pattern prior to the implementation of any additional control system. The reference scan pattern is comprised of sinusoidal waveforms with an amplitude of $10 \mu \mathrm{m}$, which yields a scan window $20 \mu \mathrm{m} \times 20 \mu \mathrm{m}$ in size. This represents the maximum scan area achievable by the MEMS nanopositioner, as determined from the device's characterization results shown in Fig. 4. Based on the nanopositioner's open-loop bandwidth, the $\mathrm{X}$ direction scanning frequency was selected as $1 \mathrm{kHz}$. Given the maximum sampling frequency of the experimental equipment and the intended image resolution ( $h \leqq 100 \mathrm{~nm}$ ), the scan window was chosen to be represented by $200 \times 200$ pixels. As a result, the frequency of the reference signal in the $\mathrm{Y}$ direction required to construct the Lissajous pattern was calculated via (3) and (4) to be $f_{y}=997.8723 \mathrm{~Hz}$, with the total scan time being $0.235 \mathrm{~s}$.

Fig. 6 shows an example of the reference signal, sensor output, and the resulting error for the nanopositioner's $\mathrm{X}$ axis during the open-loop test. Although the reference and the displacement measured by the sensor have similar amplitudes, there is a significant phase difference between the sensor output and the reference signal. This results in the error having a relatively large RMS value of $4.11 \mu \mathrm{m}$, which is $41 \%$ of the amplitude of the reference signal.

The resulting scan trajectory traversed by the nanopositioner was determined from the $\mathrm{X}$ - and Y-axis sensor outputs, and is shown in Figs. 7(a) and 7(b). It can be seen that the phase lag of the open-loop system results in a malformed Lissajous pattern, with the diagonal scan lines in particular being asymmetrically positioned with respect to the origin. In addition, the close-up view of the central section of the scan area (Fig. 7(b)) shows that the distance between adjacent scan lines is uneven, meaning that the resulting Lissajous trajectory no longer provides the effective intended resolution. Based on these results, it is therefore concluded that the implementation of a closed-loop control system is necessary to accurately track the reference sinusoidal signals and achieve high-quality AFM scans.

\section{CONTROLLER DESIGN}

As shown in Fig. 5, the open-loop response of each axis has a $40 \mathrm{~dB}$ resonance peak, which is extremely lightly damped. An open-loop step actuation results in a large overshoot as high as the steady-state change in displacement, followed by a vibration that lasts about $70 \mathrm{~ms}$, as shown in Fig. 8. This undamped behavior is not desirable when the device is intended to operate over a large range close to the displacement limits. In this case, unexpected discontinuities in the actuation, e.g., due to switching of the drive circuitry, can move the stage beyond its limits, leading to structural damage.

To alleviate the resonant response, we designed a compensator for the inner loop of the feedback control system in Fig. 9. We opted to use the following compensator:

$$
C(s)=\frac{a s}{s+b},
$$

where $a$ and $b$ are constants to be tuned. Having a zero at the origin, this type of controller, called a washout filter/ controller, ${ }^{37-40}$ is usually used in series with the plant to attenuate sensor drift and flicker noise, which are the dominant lowfrequency spurious signals for electrothermal sensors. ${ }^{15,32} \mathrm{To}$ simplify the stability analysis of the inner loop, we first approximate the plant dynamics for the $\mathrm{X}$-axis by a simple harmonic oscillator as

$$
G_{x x}(s)=\frac{c_{x}}{1+\beta_{x} s+\alpha_{x} s^{2}},
$$

where $c_{x}$ is the DC gain, $\beta_{x}=2 \zeta_{x} / \omega_{o x}$, and $\alpha_{x}=1 / \omega_{o x}^{2}$ with $\zeta_{x}$ and $\omega_{o x}$ referring to the damping ratio and natural frequency of the $\mathrm{X}$-axis, respectively. For lightly damped second-order system (8), the frequency and magnitude at the resonance peak of the frequency response are well approximated by $\omega_{o x}$ and $c_{x} / 2 \zeta_{x}$, respectively. Matching the frequency responses of the second and third order models at low frequencies and at resonance, we obtain the following 


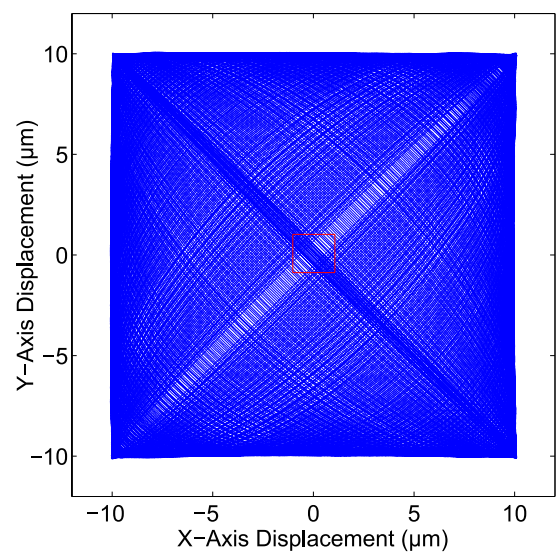

(a)

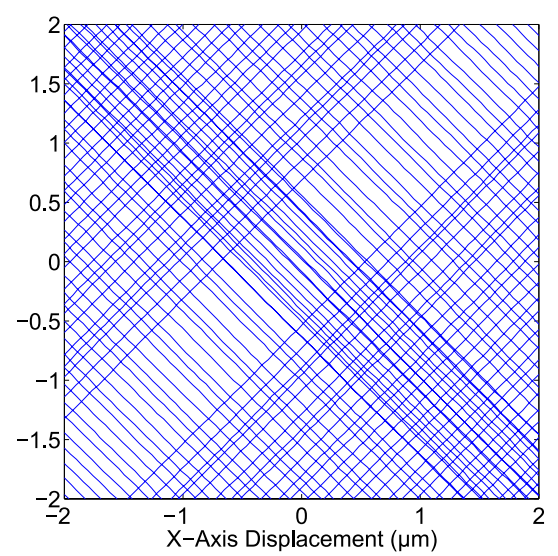

(b)

FIG. 7. (a) Open loop tracking of a $20 \mu \mathrm{m} \times 20 \mu \mathrm{m}$ Lissajous scan pattern with $f_{x}=1 \mathrm{kHz}$. (b) Close-up view of central part of scan window.

parameter values for the second-order approximations of the $\mathrm{X}$ and $\mathrm{Y}$ axes:

$$
\begin{aligned}
& c_{x}=1.604, \omega_{o x}=8000 \mathrm{rad} / \mathrm{s}, \zeta_{x}=0.0056, \\
& c_{y}=1.638, \omega_{o y}=7960 \mathrm{rad} / \mathrm{s}, \zeta_{y}=0.0065 .
\end{aligned}
$$

The frequency response of the second-order approximation for each axis is reasonably close to the experimental data within the mechanical bandwidth, as depicted in Fig. 5.

\section{A. Stability analysis of the damping loop}

Considering compensator (7) and plant models (8), the characteristic polynomial for the $\mathrm{X}$-axis after closing only the inner loop is as

$$
\alpha_{x} s^{3}+\left(\alpha_{x} b+\beta_{x}\right) s^{2}+\left(a^{\prime}+\beta_{x} b+1\right) s+b,
$$

where $a^{\prime}=c_{x} a$. Since $\alpha_{x}=1 / \omega_{o x}^{2}$ is positive, the following expressions are required to be positive by the Routh-Hurwitz stability criterion,

$$
\alpha_{x} b+\beta_{x}, \quad a^{\prime}+\beta_{x} b+1-\frac{\alpha_{x} b}{\alpha_{x} b+\beta_{x}}, \quad b .
$$

After some manipulation, the compensator parameters $a$ and $b$ should satisfy the following constraints:

$b>\max \left(0,-\frac{\beta_{x}}{\alpha_{x}}\right), \quad a>\frac{-\beta_{x}\left(\alpha_{x} \beta_{x} b^{2}+\beta_{x} b+1\right)}{\left(\alpha_{x} b+\beta_{x}\right) c_{x}}$.

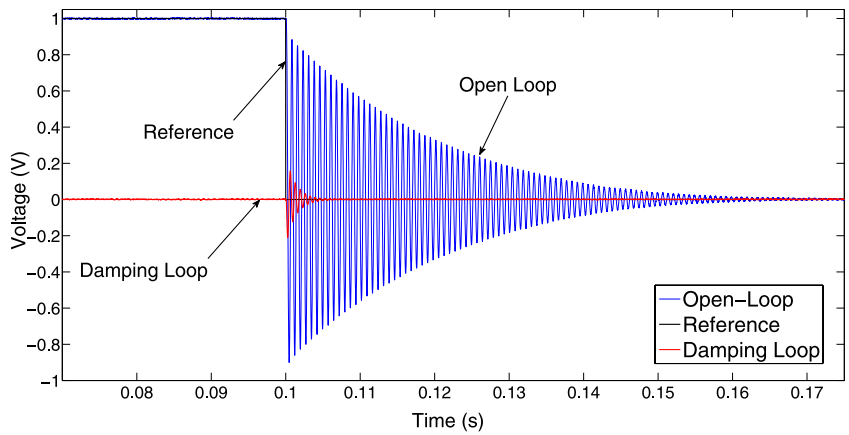

FIG. 8. Comparison between the nanopositioner step response in open-loop and damping loop conditions.
Using the parameter values for the $\mathrm{X}$-axis in (8) and the constraints in (12), we obtain the allowable compensator parameter values as indicated by the gray area in Fig. 10. An alternative way to determine a stable zone for the compensator parameters is to use the negative imaginary property of the second-order plant model. ${ }^{41}$ Due to the negative feedback in the inner loop and the zero DC gain of the washout compensator, a sufficient condition for stability of the inner loop is that $-C(s)=-a s /(s+b)$ also has a negative imaginary frequency response. This condition is simply met if the compensator parameters $a$ and $b$ are both positive, which represents a subset of the original stable zone derived by the Routh-Hurwitz method. Clearly, the negative imaginary approach is much easier to apply at the expense of not predicting a small portion of the stable zone.

Having determined the allowable ranges of compensator parameters, we searched for values in the stable zone, which provide adequate stability margins and damping for the inner loop with third-order plant model (1). The selected compensator parameter values are $a=2.4$ and $b=95000$ as indicated by the cross in Fig. 10 and which yield gain and phase margins of $18 \mathrm{~dB}$ and $44.5^{\circ}$, respectively, for the inner loop of the $\mathrm{X}$-axis. Comparing the open-loop frequency response (Fig. 5(a)) to the experimental response shown in Fig. 11 with only the inner loop closed (from input $v_{x}$ to the $\mathrm{X}$-axis output $x_{m}$ ), the selected compensator provides a large damping of about $40 \mathrm{~dB}$ at the resonance peak. The acceptable damping performance is also confirmed by the step responses in Fig. 8, where the zero DC gain of the closed-loop system further prevents the stage from exceeding the displacement limits. For the inner loop of the Y-axis, we

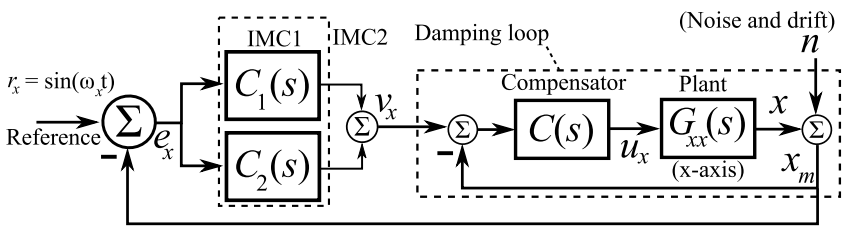

FIG. 9. Block diagram of closed-loop system. 
(a)

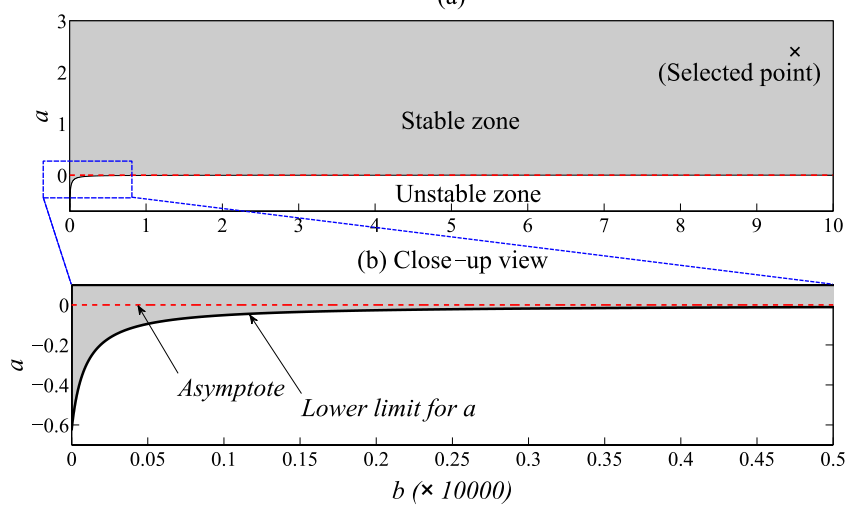

FIG. 10. The gray area shows the allowable wash-out compensator parameter values $(a, b)$ for stable operation of the inner loop. The lower limit for a is represented in (12) and the asymptote can be expressed as $-\left(\beta_{x}^{2} / c_{x}\right)\left[b+\left(1-\beta_{x}\right) / \alpha_{x}\right]$.

use the same compensator as in the $\mathrm{X}$-axis, where similar closed-loop performance is obtained.

\section{B. Internal model controllers to track sinusoid}

An efficient approach to robust tracking of a sinusoidal reference is internal model control (IMC). ${ }^{42}$ This approach incorporates controllers in the feedback loop which have eigenvalues corresponding to those of the desired references. A sinusoidal signal with frequency of $\omega_{x}=2 \pi f_{x}$ has a pair of eigenvalues on the imaginary axis at $\pm j \omega_{x}$. In the outer loop in Fig. 9, the internal model controller $C_{1}(s)=c_{1} /\left(1+(s / \omega)^{2}\right)$ (IMC1) has poles at the fundamental frequency of the Xaxis reference while $C_{2}(s)$ has poles at the second and third harmonics. Due to their undamped poles, we can also refer to these controllers as oscillators. IMC1 by itself can provide perfect tracking at steady-state, if the plant dynamics were exactly linear. However, the linearity achieved by the linear actuation mechanism is not perfect and a nonzero steadystate tracking error signal with harmonics higher than the fundamental appears if only IMC1 is used in the outer loop. In our experiments, the second and third harmonics of each reference were the most dominant components in the steadystate tracking error, when only IMC1 was used. Hence, controller $C_{2}(s)$ was designed with poles at those dominant

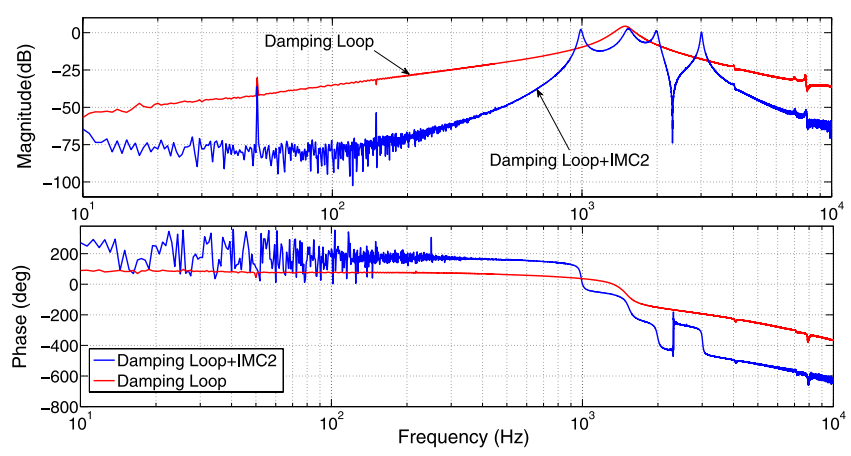

FIG. 11. The frequency responses of the damping loop and damping loop plus the internal model controllers (IMC2) for $1 \mathrm{kHz}$ in the $\mathrm{X}$ direction. harmonics to further improve the tracking performance. For the $1 \mathrm{kHz}$ sinusoidal references of the $\mathrm{X}$-axis $\left(f_{x}=1000 \mathrm{~Hz}\right)$, the tuned internal model controllers are presented as

$$
\begin{aligned}
& C_{1}(s)=\frac{c_{1}}{1+\left(\frac{s}{\omega_{x}}\right)^{2}}, \\
& C_{2}(s)=\frac{c_{2} s+c_{3}}{1+\frac{s^{2}}{\left(2 \omega_{x}\right)^{2}}}+\frac{c_{4} s+c_{5}}{1+\frac{s^{2}}{\left(3 \omega_{x}\right)^{2}}},
\end{aligned}
$$

where $c_{1}=0.28, c_{2}=c_{4}=-1.4 \times 10^{-5}$, and $c_{3}=c_{5}=0$. We used similar internal model controllers for the Y-axis, except replacing $\left(\omega_{x}\right)$ by the Y-axis reference frequency $\left(\omega_{y}\right)$. With both internal model controllers included (IMC2), the stability margins of the outer loop are $\left(3.3 \mathrm{~dB}, 35.7^{\circ}\right)$ and $(3.9 \mathrm{~dB}$, $36^{\circ}$ ) for the $\mathrm{X}$ and $\mathrm{Y}$ axes, respectively. The overall control system also maintains acceptable damping levels at both the resonance frequency and the undamped poles of the internal model controllers, as shown in Fig. 11.

The tracking performance of the closed-loop system was also assessed by having the device track the same $1 \mathrm{kHz}$ Lissajous reference signal previously described in Sec. IV. The control system was examined with both IMC1 and IMC2, where examples of the system's steady-state time-domain response are shown in Fig. 12. It is evident that the phase lag seen in the open-loop test in Fig. 6 has been eliminated, with both control implementations showing extremely good tracking of the reference sinusoidal signal. However, it is clear from the resulting error signals that the tracking accuracy is nevertheless significantly improved by the use of the higherorder oscillators in IMC2. Their inclusion causes a reduction in the RMS value of the tracking error from $0.575 \%$ of the reference amplitude to $0.104 \%$, and a similar improvement in the total harmonic distortion (THD) of the displacement signal from $0.892 \%$ to $0.110 \%$. While these tracking tests were performed, an AFM cantilever was landed on the stage to represent the experimental conditions of an actual AFM scan (refer to Sec. VI).

Regarding the tracking performance, it is therefore concluded that the higher-order harmonic oscillators in the feedback loop provide a marked improvement in the nanopositioner's tracking accuracy, and have therefore been included in the implemented control schemes for each of the Lissajous AFM imaging tests conducted at other frequencies.

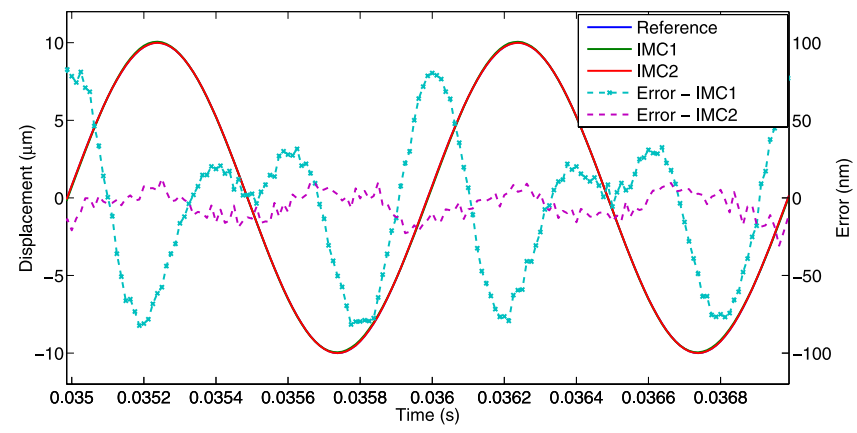

FIG. 12. Closed-loop tracking performance of the nanopositioner's X axis, using a $1 \mathrm{kHz}$ sinusoidal reference. The controller is implemented with both IMC1 and IMC2 for comparison purposes. 

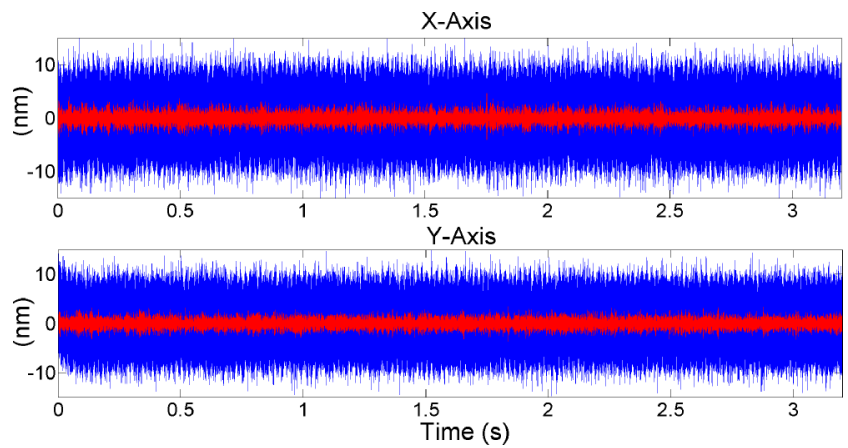

FIG. 13. The time history of the open-loop and projected noise for both directions. The blue colored and red colored signals are the open-loop and projected noise, respectively.

\section{Noise performance}

It is well understood that the steady-state tracking error contains both deterministic and stochastic components. ${ }^{26}$ The deterministic error typically originates from the insufficient bandwidth of the nanopositioner to track the reference signal and/or the uncompensated nonlinearities in the system. Here, this error is reduced by using a linear actuation mechanism and higher-order harmonic oscillators in closed loop.

The stochastic error mainly arises from the electrothermal sensors' noise, which is fed back into the system and ultimately affects the positioning accuracy of the nanopositioner. The noise component of the actual stage displacement (denoted by $x$ in Fig. 9) ${ }^{43}$ is inferred by applying the recorded open-loop noise of the electrothermal sensors to the closedloop model for $1 \mathrm{kHz}$ sinusoidal tracking (13). The time histories of the open-loop $(n)$ and the projected noise $(x)$ are depicted for both axes in Fig. 13. The RMS values of the recorded noise signal in open loop and the projected noise are reported in Table II. Approximately, the same level of projected noise was obtained with IMC2 controllers tuned for the lower different scan frequencies, as the same internal damping loop is used in each case.

The lower noise level of the projected noise can also be justified using the transfer function of the noise signal to the stage displacement

$$
T_{x n}=\frac{-\left(C_{1}(s)+C_{2}(s)+1\right) C(s) G_{x x}(s)}{1+C(s) G_{x x}(s)\left(1+C_{1}(s)+C_{2}(s)\right)},
$$

where $C_{1}(s), C_{2}(s)$, and $C(s)$ are shown in Fig. 9 and are described in (13). It can be easily shown that $T_{x n}$ rolls off either side of the frequencies of interest (i.e., the fundamental and its second and third harmonics) and therefore eliminates low and high frequency components of the noise of the electrothermal

TABLE II. RMS values of the open-loop and projected noise for both directions.

\begin{tabular}{lcc}
\hline \hline & X-axis (nm) & Y-axis (nm) \\
\hline Open loop & 3.11 & 2.99 \\
Projected & 0.96 & 0.85 \\
\hline \hline
\end{tabular}

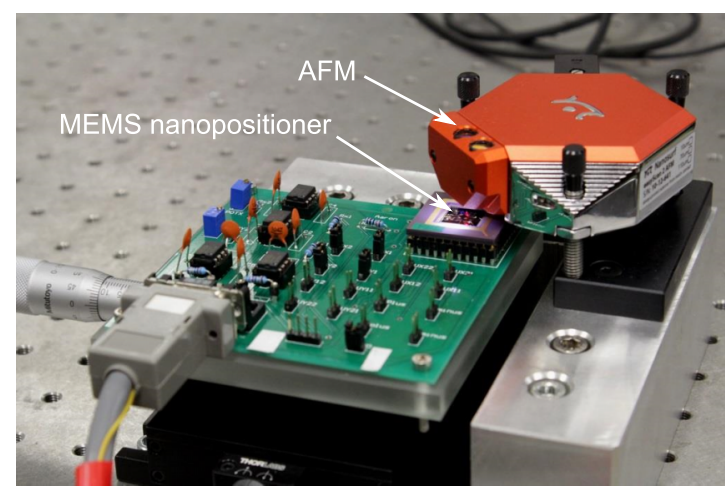

FIG. 14. The experimental setup for AFM imaging.

sensor in the actual stage displacement $(x)$, resulting in a high precision displacement.

\section{AFM IMAGING}

Following the design and testing of the closed-loop control system, a series of AFM scans were performed, with the MEMS nanopositioner being used as the in-plane scanning stage. A periodic series of gold features measuring approximately $9 \times 3 \times 0.5 \mu \mathrm{m}$ were fabricated on the surface of the nanopositioner's stage (shown in Fig. 1) to act as scan samples and facilitate testing of the AFM imaging process. As illustrated in Fig. 14, for experimental testing, the packaged and wire-bonded nanopositioner is mounted on a printed circuit board (PCB) which provides the sensing and actuation signal paths. Measurements of the sample height were obtained via the cantilever of a Nanosurf Easyscan 2 AFM, with 3D images of the sample topography being constructed from the deflection signal of the cantilever and the in-plane displacement measurements provided by the electrothermal sensors of the nanopositioner. Due to the low bandwidth of the built-in Z-axis positioner of the cantilever in this AFM, we used the constant-height contact mode. ${ }^{44}$ A contact mode cantilever with a resonance frequency of about $13 \mathrm{kHz}$ and a force constant of $0.2 \mathrm{~N} / \mathrm{m}$ is used for all experiments.

During the experiments, after closing both the damping loop and the internal model controller (IMC2) loop for each axis, the amplitudes of the reference signals for both directions were gradually increased from zero to the maximum displacement range. The Lissajous scan was then started by recording the cantilever deflection and the electrothermal sensors' data during the time span of $t_{\text {scan }}$ calculated using (5).

Given the satisfactory performance of the device when tracking a $1 \mathrm{kHz}$ Lissajous trajectory demonstrated in Sec. V B, this frequency was initially chosen for the imaging of the gold patterns. The imaging was performed using a square-shaped area of $20 \mu \mathrm{m} \times 20 \mu \mathrm{m}$ consisting of $200 \times 200$ pixels, with the data outputs being sampled at a rate of $1.28 \mathrm{MHz}$.

The obtained image is illustrated in Fig. 15. Despite the good tracking performance of the device, significant oscillations are evident in the image. As the commercial AFM 


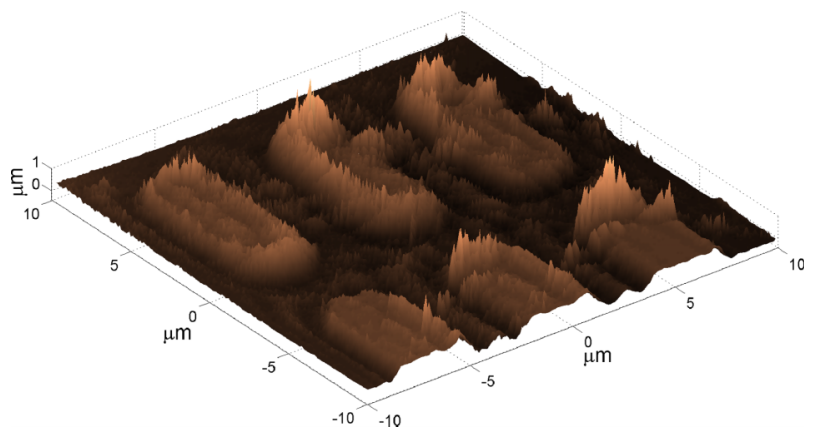

FIG. 15. The AFM image of the gold patterns obtained by a $1 \mathrm{kHz}$ Lissajous scan. Artifacts induced by the oscillation of the cantilever are visible in the image.

used in this experiment is designed for low-speed scanning purposes, the artifacts observed in the image are likely due to vibrations of the cantilever during the scan. To assess the contribution of the cantilever vibrations to the image artifacts, its dynamic properties are investigated in Sec. VI A.

\section{A. Cantilever performance}

As shown in Fig. 16, examining the time-domain cantilever deflection signal in the $1 \mathrm{kHz}$ scan reveals the presence of high-frequency vibrations in addition to the sample topography information, which explains the poor image quality of the scan image shown in Fig. 15. These vibrations have a frequency of approximately $69 \mathrm{kHz}$, and appear regardless of whether the cantilever tip is positioned on top of a gold feature or on the surface of the stage.

The origin of these vibrations was investigated by creating a finite element model of the cantilever used in the AFM scans, with the first mode shape being shown in Fig. 17. As the scans are performed in contact mode, it is assumed that the deflection of the cantilever tip remains constant while moving across a flat surface; by applying this boundary condition to the finite element model, the first resonant mode of the cantilever was found to be a bending mode at approximately $64 \mathrm{kHz}$. This vibration mode has an anti-node at the middle of the cantilever's length, which is the location where the laser spot measures its deflection. The proximity of the simulated mode's frequency to that of the observed vibrations suggests that this is the reason for their presence,

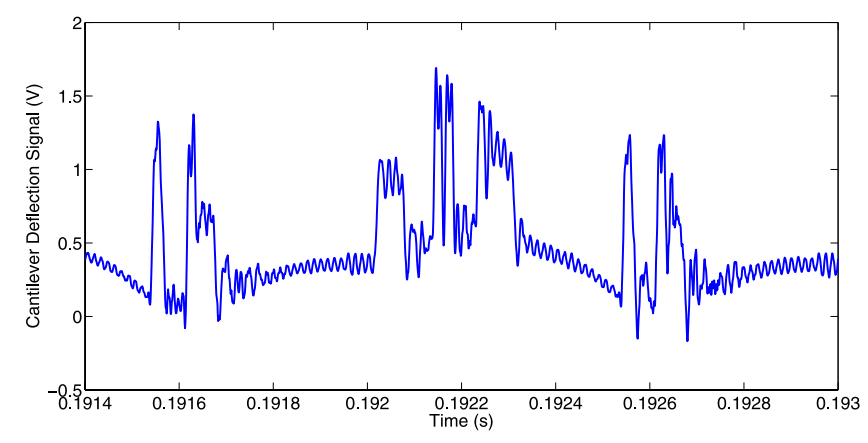

FIG. 16. High-frequency vibrations in the cantilever deflection signal during an AFM scan with reference frequency $f_{x}=1 \mathrm{kHz}$.

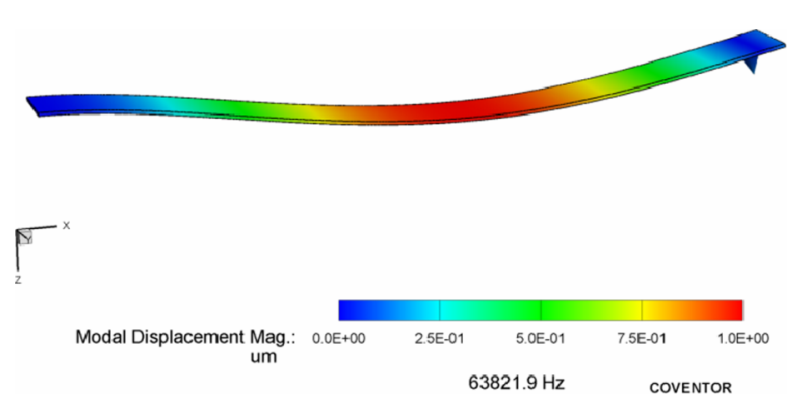

FIG. 17. First resonant mode of AFM cantilever obtained by finite element model in CoventorWare software.

however as the position of the laser beam cannot be changed, this effect cannot be rectified. Note that the FEM result shows that the vibrations are only due to the cantilever dynamics and are unrelated to the performance of the nanopositioner. In order to alleviate the cantilever oscillation and obtain AFM images with acceptable quality and less artifacts, a lower scanning frequency needs to be used. This means that while the nanopositioner is able to accurately track a Lissajous reference signal at frequencies up to $1 \mathrm{kHz}$ (as shown in Fig. 12), the cantilever is the factor limiting the effective AFM scan speed.

To rectify the cantilever's oscillation problem and therefore achieve a higher scanning rate, smaller custom-designed cantilevers with a low stiffness and high resonance frequency can be used. As an example, cantilevers with resonance frequencies between $150 \mathrm{kHz}$ and $350 \mathrm{kHz}$ for high-speed scanning are demonstrated in Ref. 9, with their small stiffness making them suitable for contact mode scanning applications. In addition, the cantilever's oscillations can be suppressed by using a constant force contact mode scanning method. This can be achieved by implementing an appropriate highbandwidth out-of-plane nanopositioner and controller for the cantilever.

\section{B. Lower frequency images}

To avoid the cantilever oscillations at high speed, the scanning frequency was lowered about four times to $260 \mathrm{~Hz}$. The same image size and pixel number are used for this scanning procedure. Fig. 18(a) illustrates the AFM image and the nanopositioner displacement signals for both axes obtained at this frequency. To estimate the fastest scanning speed before obtaining noticeable image artifacts due to cantilever vibration, the imaging was also performed at higher frequencies. In Figs. 18(b) and 18(c), the images and tracking signals for $310 \mathrm{~Hz}$ and $410 \mathrm{~Hz}$ are reported, respectively. Table III reports the parameters used to generate the internal model controllers for different scan frequencies according to (13). The Lissajous scan parameters and the RMS values of the tracking error in both axes for all scan frequencies are presented in Table IV. In this table, the equivalent raster scan frequencies are also presented, which is the required frequency of the triangular signal in a raster scan to generate an image with the same scan time and pixel number as in the Lissajous scan. As is clear in the images shown in Fig. 18, the 

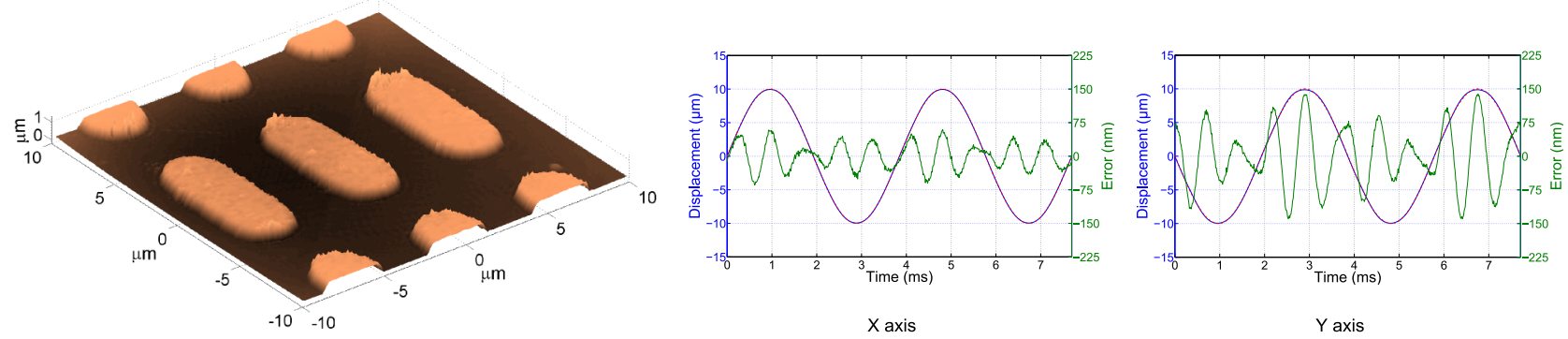

(a) $260 \mathrm{~Hz}$
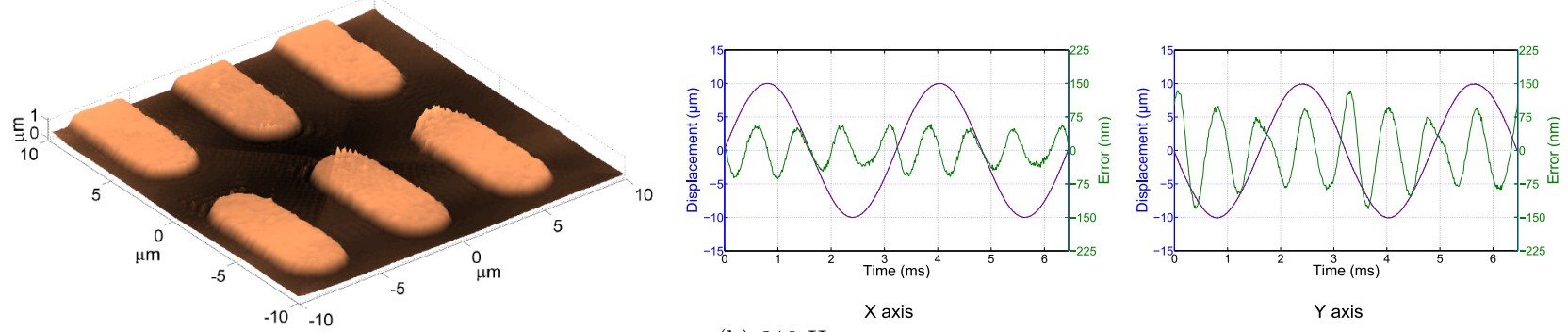

(b) $310 \mathrm{~Hz}$
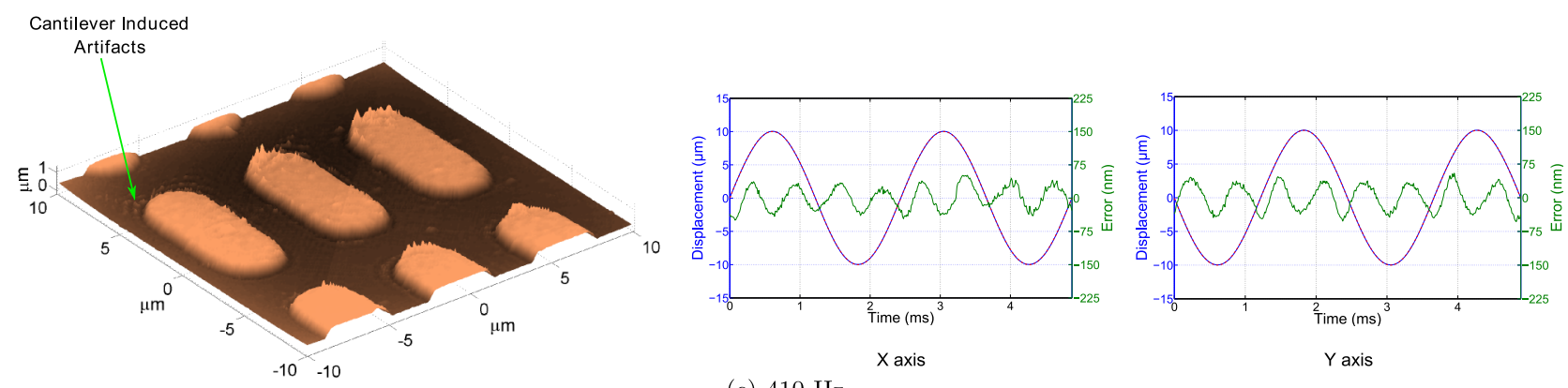

(c) $410 \mathrm{~Hz}$

FIG. 18. AFM images obtained at three different Lissajous scan frequencies. The displacements of both the $\mathrm{X}$ and $\mathrm{Y}$ axes are plotted in blue with the reference signal in red. The tracking error signals are illustrated in green. In (c), minor image artifacts induced by the cantilever vibration are highlighted.

vibration of the cantilever starts to induce minor artifacts at the scanning frequency of $410 \mathrm{~Hz}$.

By comparing the total tracking error obtained experimentally for the device (Table IV) to the sensor and the stage displacement noise level reported in Table II, it is clear that the dominant component of the tracking error is deterministic rather than stochastic. At all scan frequencies, the internal model controllers (IMC2) are designed to improve the tracking performance by suppressing the fundamental mode and the first and second harmonics in the steady-state error signal. As a result, the error signals largely consist of the 4th order and higher harmonics. These errors can be suppressed by including additional IMCs for the higher-order harmonics. However,

TABLE III. The tuned parameters and gain and phase margins for internal model controllers (IMC2) at different scan frequencies.

\begin{tabular}{llccccc}
\hline \hline $\begin{array}{l}\text { Frequency } \\
(\mathrm{Hz})\end{array}$ & $c_{1}$ & $c_{2}$ & $c_{3}$ & $c_{4}$ & $c_{5}$ & $\begin{array}{c}\text { Gain, phase } \\
\text { margin }\end{array}$ \\
\hline 260 & 7 & 0 & 0.7 & 0 & 0.28 & $4.2 \mathrm{~dB}, 49.7^{\circ}$ \\
310 & 4.2 & 0 & 0.7 & 0 & 0.21 & $2.7 \mathrm{~dB}, 38^{\circ}$ \\
410 & 2.8 & 0 & 0.35 & $5 \times 10^{-6}$ & 0 & $3.4 \mathrm{~dB}, 46^{\circ}$ \\
\hline \hline
\end{tabular}

there is a trade-off between the number of implemented IMCs and the stability of the system, as adding more controllers to cancel higher-order harmonics in the system output may lead to a reduction in the stability margins and a deterioration of the system's transient response.

\section{Discussion and future work}

The ability of the device to be used as a nanopositioner in an AFM is demonstrated in Sec. VI B. This device achieves a scanning range of $20 \mu \mathrm{m} \times 20 \mu \mathrm{m}$, which is about $40 \%$

TABLE IV. The Lissajous scan parameters, equivalent raster scan frequency, and rms errors of tracking signals for various Lissajous scan frequencies.

\begin{tabular}{lcccc}
\hline \hline$f_{x}(\mathrm{~Hz})$ & 260 & 310 & 410 & 1000 \\
\hline Scan time $\left(t_{\text {scan }}\right)$ & $0.9038 \mathrm{~s}$ & $0.7581 \mathrm{~s}$ & $0.5732 \mathrm{~s}$ & $0.2350 \mathrm{~s}$ \\
$f_{y}(\mathrm{~Hz})$ & 259.4468 & 309.3404 & 409.1277 & 997.8723 \\
Error RMS-X $(\mathrm{nm})$ & 28.6 & 34.6 & 25.1 & 10.4 \\
Error RMS-Y $(\mathrm{nm})$ & 66.8 & 68.5 & 27.9 & 8.3 \\
Raster scan & 221.3 & 263.8 & 348.9 & 851.1 \\
frequency $(\mathrm{Hz})$ & & & & \\
\hline \hline
\end{tabular}


larger than the previous nanopositioner. ${ }^{20}$ The device shows a tracking error below $70 \mathrm{~nm}$, which is less than $0.4 \%$ of the traveling range, while the previous version was demonstrated to have a higher error level of $1.3 \%$ of the displacement amplitude. ${ }^{20}$ To achieve this improved tracking performance while providing a significantly larger scan area, as explained earlier, both the deterministic and stochastic components of the error are reduced using the linear actuation mechanism, the Lissajous scan pattern, and the combination of washout and internal model controllers.

The proposed device is used for high speed scanning. We have tested the device with a maximum scanning frequency of $1000 \mathrm{~Hz}$ using the Lissajous scan method. The scan time for $20 \mu \mathrm{m} \times 20 \mu \mathrm{m}$ window size is about $0.23 \mathrm{~s}$. This scanning rate is equivalent to a raster scan with a frequency of about $851 \mathrm{~Hz}$ (Table IV), which is significantly higher than the $100 \mathrm{~Hz}$ scanning speed achieved by the previous version of the device. ${ }^{20}$

A potential drawback of this scanning method compared with conventional rastering is that the profile of the cantilever's tip has a greater effect on the quality of the obtained AFM image. While the generated image is a convolution of the topography of the sample and the cantilever's tip profile, ${ }^{45}$ in raster scanning, both the scan angle and the profile of the tip with respect to the sample remain constant. This is no longer true for Lissajous scanning due to the shape of the cyclical path taken by the cantilever, meaning that the broadening of features across the generated image may occur in a possibly irregular manner.

One possible solution to this issue is the use of carbon nanotubes as the scanning probe ${ }^{46}$ rather than conventional silicon or silicon nitride tips. These conventional tips are typically pyramidal in shape, with a cone angle of $20^{\circ}-30^{\circ}$ and a radius of curvature of several tens of nanometers. ${ }^{47}$ In contrast, single walled carbon nanotubes are cylindrical in shape and have a radius below $2.5 \mathrm{~nm}$, meaning that the effect of feature broadening within the generated AFM image is likely to be significantly reduced and is much less dependent on the changing scan angle throughout the Lissajous path.

A further potential issue is that the constantly changing scan angle results in a torque being applied to the cantilever in all directions throughout the Lissajous scan. This may again affect the quality of the obtained image in a nonuniform manner. However, this effect may be reduced by performing the AFM scan in tapping mode rather than contact mode, significantly reducing the lateral forces on the cantilever. ${ }^{8}$

\section{CONCLUSIONS}

A new 2-DOF parallel kinematic MEMS nanopositioner with bidirectional actuation capability is presented, with the device being applicable for use in an on-chip AFM. The nanopositioner features a mechanical bandwidth of about $1.3 \mathrm{kHz}$ and a stroke of more than $\pm 10 \mu \mathrm{m}$ in both orthogonal axes. A linear actuation mechanism is implemented for both axes, and the static and dynamic behavior of the device are characterized in order to implement the controller. To measure the stage displacement in both axes, electrothermal sensors are used with a transimpedance readout circuit. The highly resonant behavior of the device is damped using a washout controller in the inner controller loop, together with IMCs in an outer loop to track a Lissajous trajectory. This is the first implementation of the Lissajous scan approach for MEMS-based nanopositioning, which allows a higher scanning frequency to be achieved. The nanopositioner is used to operate as part of an AFM, performing high speed Lissajous scans with frequencies up to $1 \mathrm{kHz}$. The results show that the device is a valuable nanopositioning platform for micro and nanoscale applications, especially in on-chip video-rate atomic force microscopy.

\section{ACKNOWLEDGMENTS}

This work was supported by the Australian Research Council and the University of Newcastle, Australia.

${ }^{1}$ J. J. Gorman, Y.-S. Kim, A. E. Vladár, and N. G. Dagalakis, Int. J. Nanomanuf. 1, 710 (2007).

${ }^{2}$ J. J. Gorman, N. G. Dagalakis, and B. G. Boone, "Free-space laser communication and active laser illumination III," Proc. SPIE 5160, 170-181 (2004).

${ }^{3}$ J. Shi, J. Dertouzos, A. Gafni, and D. Steel, in Fluorescence Spectroscopy, Methods in Enzymology Vol. 450, edited by L. Brand and M. L. Johnson (Academic Press, 2008), pp. 129-157.

${ }^{4}$ H. Lan, Y. Ding, H. Liu, and B. Lu, Microelectron. Eng. 84, 684 (2007).

${ }^{5}$ S. M. Salapaka and M. V. Salapaka, IEEE Control Syst. Mag. 28, 65 (2008).

${ }^{6}$ G. Binnig, C. F. Quate, and C. Gerber, Phys. Rev. Lett. 56, 930 (1986).

${ }^{7}$ Y. K. Yong, S. O. R. Moheimani, B. J. Kenton, and K. K. Leang, Rev. Sci. Instrum. 83, 121101 (2012).

${ }^{8}$ M. W. Fairbairn and S. O. R. Moheimani, IEEE Control Syst. Mag. 33, 46 (2013).

${ }^{9}$ G. E. Fantner, G. Schitter, J. H. Kindt, T. Ivanov, K. Ivanova, R. Patel, N. Holten-Andersen, J. Adams, P. J. Thurner, I. W. Rangelow, and P. K. Hansma, in Proceedings of the Seventh International Conference on Scanning Probe Microscopy, Sensors and Nanostructures [Ultramicroscopy 106, 881 (2006)].

${ }^{10}$ L. L. Chu and Y. B. Gianchandani, J. Micromech. Microeng. 13, 279 (2003).

${ }^{11}$ J. J. Gorman, Y.-S. Kim, and N. G. Dagalakis, in 2006 ASME International Mechanical Engineering Congress and Exposition (ASME, Chicago, USA, 2006), pp. 1-9.

${ }^{12}$ D. Mukhopadhyay, J. Dong, E. Pengwang, and P. Ferreira, Sens. Actuators, A 147, 340 (2008).

${ }^{13}$ D. M. Brouwer, B. R. de Jong, and H. M. J. R. Soemers, Precis. Eng. 34, 307 (2010).

${ }^{14}$ S. Bergna, J. J. Gorman, and N. G. Dagalakis, in 2005 ASME International Mechanical Engineering Congress and Exposition (ASME, Orlando, USA, 2005), pp. 1-8.

${ }^{15}$ Y. Zhu, A. Bazaei, S. O. R. Moheimani, and M. R. Yuce, J. Microelectromech. Syst. 20, 711 (2011).

${ }^{16}$ E. Eleftheriou, T. Antonakopoulos, G. K. Binnig, G. Cherubini, M. Despont, A. Dholakia, U. Durig, M. A. Lantz, H. Pozidis, H. E. Rothuizen, and P. Vettiger, IEEE Trans. Magn. 39, 938 (2003).

${ }^{17}$ A. Pantazi, M. A. Lantz, G. Cherubini, H. Pozidis, and E. Eleftheriou, Nanotechnology 15, S612 (2004).

${ }^{18}$ A. Pantazi, A. Sebastian, G. Cherubini, M. Lantz, H. Pozidis, H. Rothuizen, and E. Eleftheriou, IEEE Trans. Control Syst. Technol. 15, 824 (2007).

${ }^{19}$ A. G. Fowler, A. Laskovski, A. C. Hammond, and S. O. R. Moheimani, J. Microelectromech. Syst. 21, 771 (2012).

${ }^{20}$ A. Mohammadi, A. G. Fowler, Y. K. Yong, and S. O. R. Moheimani, J. Microelectromech. Syst. 23, 610 (2014).

${ }^{21}$ R. Hickey, D. Sameoto, T. Hubbard, and M. Kujath, J. Micromech. Microeng. 13, 40 (2003).

${ }^{22}$ N. B. Hubbard, M. L. Culpepper, and L. L. Howell, Appl. Mech. Rev. 59, 324 (2006).

${ }^{23} \mathrm{M}$. Maroufi and S. O. R. Moheimani, in IEEE/ASME International Conference on Advanced Intelligent Mechatronics (IEEE, Wollongong, Australia, 2013), pp. 335-340.

${ }^{24}$ A. Sebastian and D. Wiesmann, J. Microelectromech. Syst. 17, 911 (2008). 
${ }^{25}$ A. Bazaei, Y. K. Yong, and S. O. R. Moheimani, Rev. Sci. Instrum. 83, 063701 (2012).

${ }^{26}$ T. Tuma, A. Sebastian, J. Lygeros, and A. Pantazi, IEEE Control Syst. Mag. 33, 68 (2013).

${ }^{27}$ Y. K. Yong, A. Bazaei, and S. O. R. Moheimani, IEEE Trans. Nanotechnol. 13, 85 (2014).

${ }^{28}$ I. A. Mahmood and S. O. R. Moheimani, Nanotechnology 20, 365503 (2009).

${ }^{29}$ Y. K. Yong, S. O. R. Moheimani, and I. R. Petersen, Nanotechnology 21, 365503 (2010).

${ }^{30}$ A. Cowen, G. Hames, D. Monk, S. Wilcenski, and B. Hardy, SOIMUMPS Design Handbook (MEMSCAP Inc., 2011).

${ }^{31}$ R. Legtenberg, A. W. Groeneveld, and M. Elwenspoek, J. Micromech. Microeng. 6, 320 (1996).

${ }^{32}$ A. G. Fowler, A. Bazaei, and S. O. R. Moheimani, J. Microelectromech. Syst. 22, 687 (2013).

${ }^{33}$ A. Mohammadi, M. R. Yuce, and S. O. R. Moheimani, J. Microelectromech. Syst. 21, 1279 (2012)

${ }^{34}$ Y. Yong, S. Aphale, and S. O. R. Moheimani, IEEE Trans. Nanotechnol. 8, 46 (2009).

${ }^{35}$ M. A. Soderstrand, G. Berchin, and R. S. Roberts, Int. J. Electron. 78, 25 (1995).
${ }^{36}$ S. P. Wadikhaye, Y. K. Yong, B. Bhikkaji, and S. O. R. Moheimani, Smart Mater. Struct. 23, 025030 (2014).

${ }^{37}$ M. Hassouneh, H.-C. Lee, and E. Abed, in Proceedings of the 2004 American Control Conference (IEEE, Boston, USA, 2004), Vol. 5, pp. 3950-3955.

${ }^{38} \mathrm{~W}$. Hori and T. Takimoto, in 2011 IEEE/SICE International Symposium on System Integration (SII) (IEEE, Kyoto, Japan, 2011), pp. 491-496.

${ }^{39}$ A. Bazanella, P. Kokotovic, and A. E. Silva, Int. J. Control 73, 600 (2000).

${ }^{40} \mathrm{~T}$. Takimoto and S. Yamamoto, in 2007 American Control Conference (IEEE, New York, USA, 2007), pp. 5390-5393.

${ }^{41}$ A. Lanzon and I. Petersen, IEEE Trans. Autom. Control 53, 1042 (2008).

${ }^{42}$ B. Francis and W. Wonham, Automatica 12, 457 (1976).

${ }^{43}$ A. Bazaei, Y. Yong, S. O. R. Moheimani, and A. Sebastian, IEEE Trans. Control Syst. Technol. 20, 453 (2012).

${ }^{44}$ Q. Zou, K. Leang, E. Sadoun, M. Reed, and S. Devasia, Asian J. Control 6, 164 (2004).

${ }^{45}$ G. Reiss, F. Schneider, J. Vancea, and H. Hoffmann, Appl. Phys. Lett. 57, 867 (1990).

${ }^{46}$ L. Chen, C. L. Cheung, P. D. Ashby, and C. M. Lieber, Nano Lett. 4, 1725 (2004).

${ }^{47}$ J. H. Hafner, C. L. Cheung, A. T. Woolley, and C. M. Lieber, Prog. Biophys. Mol. Biol. 77, 73 (2001). 